\section{(ce \\ We are now pursuing both autonomous thermo- regulatory technologies and adaptive camouflage systems that function in the visible}

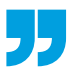

\title{
SOFT ROBOTICS
}

\section{Hiding in reflections}

Cephalopods, such as squids and octopuses, change the colour and pattern of their skin to communicate between themselves and to hide from predators. This colour-changing ability is enabled by their complex skin, which contains pigmented cells as well as cells that reflect light.

Now, writing in Science, Alon Gorodetsky and colleagues report an adaptive system that reflects infrared light, and hence also heat, in a similar way to how the squid's skin reflects visible light. The active layer in their centimetre-scale devices is either mechanically or electrically actuated, resulting in a change from a wrinkled to a smooth surface, which is accompanied by a change in the reflectance of infrared light. "We were inspired by both science fiction and science fact, in particular, seeing fictional dinosaurs disappear and reappear when visualized with an infrared camera in Jurassic World, and seeing squids behave similarly when visualized with a standard camera underwater," says Gorodetsky.

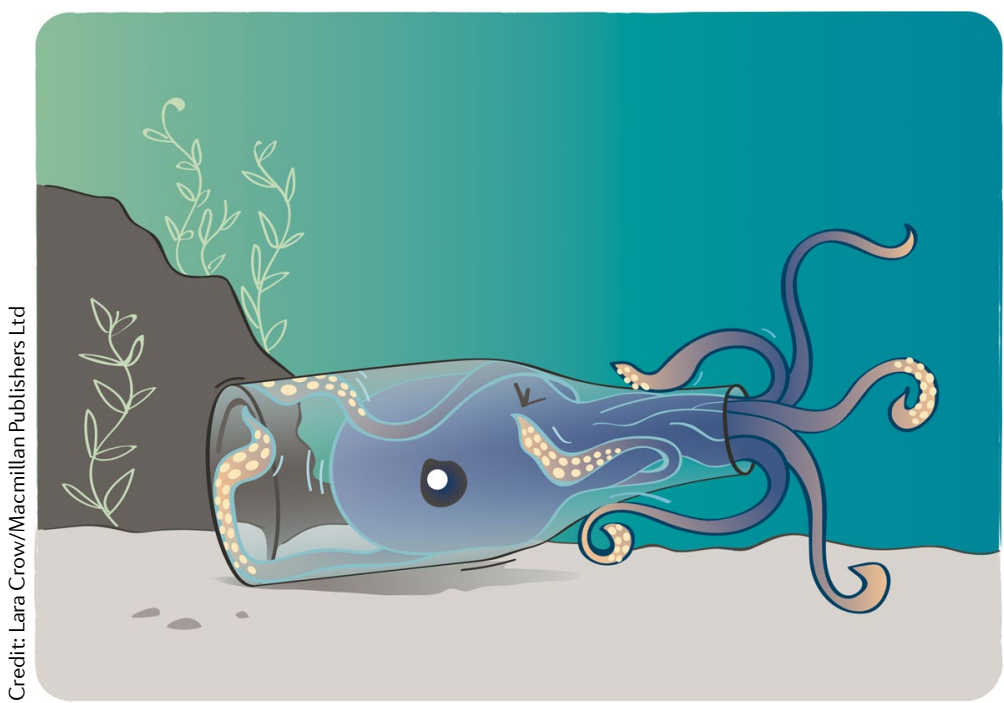

The electroactive layer of the devices is a dielectric elastomer membrane. Polymeric protonconducting electrodes are placed above and below this layer, and then the surface of the top electrode is modified with an infrared-reflecting coating. Initially, the devices are mechanically contracted to release tension in the different components, which causes micrometre-scale wrinkling of the infrared-reflecting coating. Upon the application of an external stimulus - either mechanical strain or an electrical field - the infrared-reflecting coating goes from wrinkled to smooth, changing the way the devices reflect infrared light. types of reflective devices - for broadband and narrowband infrared reflecting applications. For broadband devices, a thin film of aluminium metal was deposited on the top electrode to form the infrared-reflecting coating. For narrowband devices, alternating layers of titanium dioxide and
The researchers developed two silicon dioxide formed the coating. Both types of devices are fully reversible, stable over many cycles and give reproducible results. Moreover, the aluminium-coated devices can operate autonomously when connected to a temperature sensor.

The researchers demonstrate the functionality of their infrared-reflecting broadband system by placing a squidshaped device on a warm surface $\left(\sim 35^{\circ} \mathrm{C}\right)$ and imaging it with an infrared camera. In the absence of actuation, the squid-shaped device is indistinguishable from the background when visualized with the infrared camera. On application of an electrical stimulus to the device, the device's active area expands and the silhouette of the squid can be seen by the infrared camera. Although the actual temperature remains constant, the increase in reflectance makes the device appear $\sim 2{ }^{\circ} \mathrm{C}$ warmer than the background.

The potential applications of adaptive infrared-reflecting systems are numerous and include building insulation elements, energy-conserving windows, components for spacecraft, wearable thermoregulatory materials and camouflage platforms. The researchers are keen to enhance the performance of the infrared-reflecting system. "We are now pursuing both autonomous thermoregulatory technologies and adaptive camouflage systems that function in the visible," concludes Gorodetsky.

Maria Longobardi, Associate Editor, Nature Reviews Physics

ORIGINAL ARTICLE Xu, C. et al. Adaptive infrared-reflecting systems inspired by cephalopods. Science 359, 1495-1500 (2018) 